\title{
Efficient Magnetization Reversal with Noisy Currents
}

\author{
Wouter Wetzels, Gerrit E. W. Bauer, and Oleg N. Jouravlev* \\ Kavli Institute of NanoScience, Delft University of Technology, Lorentzweg 1, 2628 CJ Delft, The Netherlands
}

(Received 16 November 2005; published 30 March 2006)

\begin{abstract}
We propose to accelerate reversal of the ferromagnetic order parameter in spin valves by electronic noise. By solving the stochastic equations of motion we show that the current-induced magnetization switching time is drastically reduced by a modest level of externally generated current (voltage) noise. This also leads to a significantly lower power consumption for the switching process.
\end{abstract}

PACS numbers: 75.60.Jk, 72.70.+m, 75.75.+a

The dynamics of the ferromagnetic order parameter persists to pose a challenging problem of fundamental and applied nature [1]. With increasing bit density of mass data storage devices and emergence of the magnetic random access memory (MRAM) concept, the speed and energy dissipation of the magnetization switching process have become important issues. In the present MRAM generation, magnetic bits are written by spatially extended Ørsted magnetic fields, which sets limits to bit size and power consumption. An attractive alternative method is the current-induced magnetization switching predicted by theoreticians $[2,3]$ and confirmed experimentally in nanopillar devices [4-6]. In these spin valves, which consist basically of an electrically connected ferromagnetic-normal-ferromagnetic metals sandwich, the electric current is polarized in a "fixed" layer of high magnetic coercivity and exerts a "spin-transfer torque" on the second magnetically soft ferromagnet as sketched in Fig. 1. Recently, time-resolved measurements of the current-induced magnetization dynamics have been reported [7]. Advanced theoretical models for the spin and charge transport in magnetic devices [8-10] lead to a reasonable description of the magnetization dynamics within the macro-spin model, in which the magnetization is assumed to move rigidly under external magnetic field and spin-transfer torques [11,12]. We should note that in larger devices evidence has been found for spin waves and more complicated excitations that require full micromagnetic simulations [13].

The main obstacle that prevents wide application of current-induced switching is the high critical current needed to reverse the magnetization. There are proposals on how to reduce the critical current by sample design [12] and optimizing the switching process by a precessional switching strategy [14]. Since the spin-transfer torque vanishes for the collinear stable point of a spin valve, the switching time depends strongly on processes that induce a canting between the magnetizations such that the spintransfer torque starts to kick in. This happens, for example, by increasing temperature, and is the basic idea of the precharging strategy by Devolder et al. [15]. More advanced strategies used in conventional magnetization switching require pulse-shaped microwaves [16] and rely on precise knowledge of the magnetization dynamics with proper feedback.

Electrical and magnetization noise usually degrades device and system performance and often efforts have to be undertaken to reduce it as much as possible. In nonlinear systems, intentionally added noise may, e.g., enhance the quality of signal transmission by the phenomenon of stochastic resonance. Noise generators find useful application, for example, to test the response of a system to noise, to generate random signals for use in encryption or to minimize the effect of quantization errors by a method called dithering. In this Letter, we propose a simple method to improve the energy efficiency of current-induced magnetization switching by adding noise to the electric circuit connected to the device. We demonstrate that this leads to a reversal process with increased switching speed and less energy dissipation.

The magnetization dynamics in the macrospin model for a small magnetic grain is described by the LandauLifshitz-Gilbert (LLG) equation [17]

$$
\dot{\boldsymbol{m}}=-\gamma \mathbf{m} \times \boldsymbol{H}_{\mathrm{eff}}+\alpha \mathbf{m} \times \dot{\mathbf{m}},
$$

where $\gamma$ is the gyromagnetic ratio and $\alpha$ the Gilbert damping constant. The magnetization direction $\mathbf{m}=$ $(\cos \varphi \sin \theta, \sin \varphi \sin \theta, \cos \theta)$ is parameterized by the angles $\theta$ and $\varphi$, and we consider switching between $\theta=0$ (magnetization pointing up) and $\theta=\pi$ (down). When a current bias is applied to the device in Fig. 1, the LLG equation for

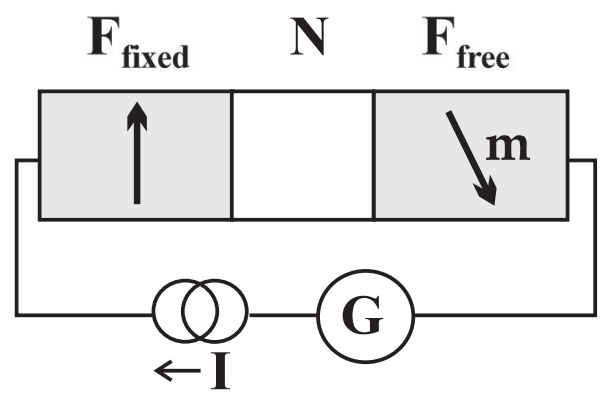

FIG. 1. Schematic picture of the spin valve under consideration. The applied potential on the left/right side is $\pm V / 2$. The charge current $I$ flows clockwise and $G$ is a noise generator. 
the free layer $F_{\text {free }}$ needs to be modified to include the spintransfer torque $\dot{\boldsymbol{m}}_{\text {torque }}=-\left(\gamma / \mathcal{M}_{S}\right) \mathbf{m} \times \mathbf{I}_{\text {free }} \times \mathbf{m}$, where $\mathcal{M}_{S}$ is the total magnetic moment of the ferromagnet. The electric charge and spin currents driven through the system fluctuate due to thermal and shot noise [18]. Here we wish to investigate the effect of intentionally applied current fluctuations with spectral density and bandwidth controlled by an external noise generator. The fluctuating (spin) current through the interfaces of $F_{\text {free }}$ creates a fluctuating spin torque that can be taken into account by adding a fluctuating torque to the LLG equation, $\dot{\boldsymbol{m}}_{\text {fluct }} \equiv \mathbf{m} \times(\mathbf{h} \times \mathbf{m})$, where we introduced an effective random field $\mathbf{h}=h_{\theta} \hat{\boldsymbol{\theta}}+$ $h_{\phi} \hat{\boldsymbol{\phi}}+h_{r} \hat{\mathbf{r}}$ in spherical coordinates, with $\left\langle h_{i}(t)\right\rangle=0$ and $\left\langle h_{i}(t) h_{j}\left(t^{\prime}\right)\right\rangle=\mu_{i j} \delta_{i j}\left(t-t^{\prime}\right)$ for $i, j \in\{\theta, \phi, r\}$. Such a model befits an electromagnetic environment with a large number of degrees of freedom that generates Gaussian noise with correlation times much shorter than the magnetization response time. At room temperature, shot noise does not have to be taken into account [18]. The LLG equation in the presence of random magnetic fields leads to a stochastic equation for the three components of the unit vector $\boldsymbol{m}$, which can be reduced to the Fokker-Planck equation [17]. This allows us to access the magnetization reversal time as a function of applied switching current and current noise.

The switchable ferromagnet is assumed to be a prolate spheroid with easy axis along $z$, i.e., $\mathbf{H}_{\text {eff }}=H_{A} \cos \theta \hat{z}$ and an eventual uniaxial crystal anisotropy with the same symmetry. For thermal stability of the equilibrium magnetization the ratio of the height of the effective energy barrier and the thermal energy should be high, i.e., $H_{A} \mathcal{M}_{S} \gg$ $k_{B} T$. For a magnet with a combination of easy-plane and uniaxial anisotropy, the switching times behave very similarly after taking into account that the weaker anisotropy governs the energy of the saddle point [11].

We use the formalism developed in [8] to find the charge and spin currents through the spin valve. The ferromagnetnormal metal contacts are assumed to have identical spindependent conductances $g^{\Uparrow \Uparrow}$ and $g^{\Downarrow}$ (in units of the conductance quantum $\left.e^{2} / h\right)$ with $p=\left(g^{\Uparrow}-g^{\downarrow}\right) /\left(g^{\Uparrow}+g^{\Downarrow}\right)$. We approximate the mixing conductance $g^{\Uparrow \downarrow}$, the interfacespecific parameter for the spin-transfer torque, by $(g \Uparrow+$ $g \downarrow \downarrow) / 2$, which is sufficiently accurate for our purposes. The spin accumulation in the normal metal in quasiequilibrium can be found by using current conservation and spin conservation in the normal metal. Spin flip in the normal metal is disregarded, while that in the ferromagnet is absorbed in the spin-dependent conductances. Under an applied voltage bias $V$, we then find a charge current

$$
\frac{I}{e V}=\frac{e}{2 h}\left(g^{\Uparrow}+g^{\Downarrow}\right)\left(1-p^{2} \sin ^{2} \frac{\theta}{2}\right),
$$

and a spin current leaving the free ferromagnetic layer for the normal metal node

$$
\frac{\mathbf{I}_{\text {free }}}{e V}=\frac{g^{\Uparrow}-g^{\Downarrow}}{16 \pi}\left(-2 \mathbf{m} \cos ^{2} \frac{\theta}{2}+\hat{\boldsymbol{\theta}} \sin \theta\right) .
$$

By inserting this expression into $\dot{\boldsymbol{m}}_{\text {torque }}$, we find for the spin torque contribution to the dynamics

$$
\dot{\boldsymbol{m}}_{\text {torque }}=\gamma J_{S} \sin \theta \hat{\boldsymbol{\theta}}, \quad J_{S}=\left(g^{\Uparrow}-g^{\Downarrow}\right) e V /\left(16 \pi \mathcal{M}_{S}\right) .
$$

Since this torque vanishes at $\theta=0, \pi$, the absence of (temperature-induced) fluctuations would imply an infinite switching time. Combining all contributions discussed above, and disregarding an angle dependence of the Gilbert damping due to spin pumping [19], the LLG equation reduces to

$$
\begin{aligned}
& \dot{\theta}=-\gamma \alpha H_{A} \cos \theta \sin \theta-\gamma J_{S} \sin \theta+h_{\theta}, \\
& \dot{\varphi}=\gamma H_{A} \cos \theta+\alpha \gamma J_{S} \sin \theta+h_{\varphi} / \sin \theta .
\end{aligned}
$$

In the following we focus on the effect of current fluctuations $\Delta I(t)=I(t)-\langle I\rangle$ with spectrum

$$
S(\omega)=\int d t e^{i \omega t}\langle\Delta I(t) \Delta I(0)\rangle,
$$

that are generated externally on top of the thermal noise. The noise spectral density is taken to be a constant $S_{w}$ over a bandwidth $\Delta \omega$ that depends on the typical time scales of the system: the system is not sensitive to fluctuations slower than the time scale of the magnetization dynamics (the inverse Larmor frequency) or faster than the electron transfer time defined by voltage. Let us define the fluctuations of spin currents (where $\sigma=\uparrow, \downarrow$ for a chosen quantization axis), $\Delta I^{\sigma}(t)=I^{\sigma}(t)-\left\langle I^{\sigma}\right\rangle$ and the corresponding noise power

$$
S^{\sigma \sigma^{\prime}}=\frac{1}{2}\left\langle\Delta I^{\sigma}(t) \Delta I^{\sigma^{\prime}}\left(t^{\prime}\right)+\Delta I^{\sigma^{\prime}}\left(t^{\prime}\right) \Delta I^{\sigma}(t)\right\rangle .
$$

Since $I(t)=I^{\dagger}(t)+I^{\downarrow}(t)$, the charge noise power can be written as $S^{\mathrm{ch}}=\sum_{\sigma, \sigma^{\prime}=\uparrow, \downarrow} S^{\sigma \sigma^{\prime}}$. The spin current polarized perpendicular to $\mathbf{m}$ transfers angular momentum to the ferromagnet almost instantaneously at the interface. Taking the spin quantization axis in the direction of $\hat{\boldsymbol{\theta}}$ i.e., perpendicular to the magnetization of $F_{\text {free }}$, there is therefore no correlation between the (transverse) spin currents $I_{r}^{\sigma}(t)$ on both sides $(r=L, R)$ of $F_{\text {free }}$. The component of the field $\mathbf{h}$ relevant for our discussion is $h_{\theta}$, transverse to the magnetization, and can be expressed as $h_{\theta}(t)=\sum_{r=L, R}\left(\gamma \hbar / 2 e \mathcal{M}_{S}\right)\left[I_{r}^{\dagger}(t)-I_{r}^{\downarrow}(t)\right]$.

We describe the switching process in the Néel-Brown model of thermally assisted magnetization reversal [17]. We consider a statistical ensemble of particles on the unit sphere that represents the probability density for the magnetization direction. Under the influence of the (random) forces these particles diffuse over the sphere. A FokkerPlanck equation describes the evolution of the probability density $P(\theta, \varphi, r)$ to find the magnetization vector in a certain direction $[11,17]$.

$$
\begin{aligned}
\frac{\partial P}{\partial t}= & -\frac{\partial}{\partial \theta} A_{\theta} P+\frac{1}{2} \frac{\partial^{2}}{\partial \theta^{2}} B_{\theta \theta} P-\frac{\partial}{\partial \varphi} A_{\varphi} P \\
& +\frac{1}{2} \frac{\partial^{2}}{\partial \varphi^{2}} B_{\varphi \varphi} P
\end{aligned}
$$


with $A_{\theta}=-\gamma \alpha H_{A} \cos \theta \sin \theta-\gamma J_{S} \sin \theta+\frac{1}{2} \mu_{\theta \theta} \cot \theta$, $B_{\theta \theta}=\mu_{\theta \theta}, \quad A_{\varphi}=\gamma H_{A} \cos \theta+\alpha \gamma J_{S} \sin \theta$, and $B_{\varphi \varphi}=$ $\mu_{\varphi \varphi} / \sin ^{2} \theta$. The diffusion constant $\mu_{\theta \theta}$ is affected by the noise power $S_{w}$. We consider the limit of small polarization $p$ in which $\mu_{\theta \theta}$ becomes independent of $\theta$. The value of $\mu_{\varphi \varphi}$ is then equal to $\mu_{\theta \theta}$, but is not relevant for the calculation of the switching time. The new diffusion parameter can then be written in terms of $S_{w}$ :

$$
\mu_{\theta \theta}=\mu_{\text {thermal }}+\frac{1}{2}\left(\frac{\gamma \hbar}{e \mathcal{M}_{S}}\right)^{2} S_{w},
$$

where

$$
\mu_{\text {thermal }}=\frac{2 \gamma \alpha k_{B} T}{\mathcal{M}_{S}}
$$

is the Néel-Brown diffusion constant.

We introduce the surface probability current $\mathbf{j}(\theta, \varphi)$ defined as $\partial W / \partial t=-\boldsymbol{\nabla} \cdot \mathbf{j}$ in terms of the surface probability density $W(\theta, \varphi)=P(\theta, \varphi) / \sin \theta$. In the steady state

$$
\mathbf{j} \cdot \hat{\boldsymbol{\theta}}=A_{\theta} W-\frac{1}{2 \sin \theta} \frac{\partial}{\partial \theta} B_{\theta \theta} W \sin \theta \equiv 0 .
$$

Since $W$ does not depend on $\varphi$,

$$
W(\theta) \propto w(\theta)=\exp \frac{-\gamma \alpha H_{A} \sin ^{2} \theta+2 \gamma J_{S} \cos \theta}{\mu_{\theta \theta}} .
$$

In Fig. 2 the probability density is shown for some combinations of current and noise power. It can be seen that applying a current results in an asymmetric distribution because the spin torque drives the magnetization to the second well, whereas an increased noise power broadens the distribution in the wells.

For high barriers, Kramer's method can be applied to find the switching time of the magnet. In that limit equilibrium is attained separately in the regions $0 \leq \theta \leq \theta_{1}$ and $\theta_{2} \leq \theta \leq \pi$ (potential well 1 and 2). The effective potentials at $\theta_{1}$ and $\theta_{2}$ should be separated from the potential minimum by several thermal energies $k_{B} T$ to ensure that the probability to find the magnetization in the middle region is low. The probability current $I_{m}$ in this middle region defined as $\mathbf{j} \cdot \hat{\boldsymbol{\theta}}=I_{m} /(2 \pi \sin \theta)$, is then small and does not disturb the equilibrium distribution in the two wells significantly. In the region $\theta_{1} \leq \theta \leq \theta_{2}$ (see Fig. 2) then

$$
\dot{n}_{1}=-\dot{n}_{2}=-\frac{n_{1}}{\tau_{1}}+\frac{n_{2}}{\tau_{2}}=-I_{m},
$$

where $n_{\mathrm{s}}$ is the probability to be in well $\mathrm{s}=1,2$. The escape time from well 1 ,

$$
\tau_{1}=\frac{2}{\mu_{\theta \theta}} \int_{0}^{\theta_{1}} d \theta w(\theta) \sin \theta \int_{\theta_{1}}^{\theta_{2}} d \xi[w(\xi) \sin \xi]^{-1}
$$

is a good estimate for the switching time. When $2 \gamma \alpha H_{A} / \mu_{D},\left|\alpha H_{A} / J_{S}\right| \gg 1$, we can calculate the integrals using Taylor expansions up to second order in $\theta$ and approximating $\sin \theta$ by $\theta$ (Ref. [17]):

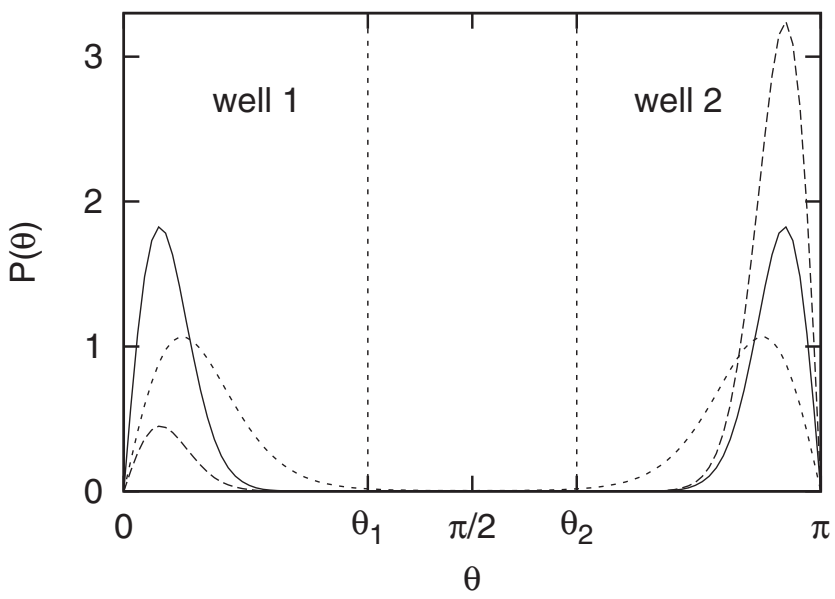

FIG. 2. The probability density for the magnetization direction in the steady state as a function of the angle $\theta$. The density in the absence of a current is shown for a noise spectral density of $S_{w}=3.0 \times 10^{-20} \mathrm{C}^{2} \mathrm{~s}^{-1}$ (solid) and $S_{w}=1.0 \times$ $10^{-19} \mathrm{C}^{2} \mathrm{~s}^{-1}$ (dotted). The asymmetry in the dashed curve is caused by a current of $0.1 \mathrm{~mA}$ with noise spectral density $S_{w}=$ $3.0 \times 10^{-20} \mathrm{C}^{2} \mathrm{~s}^{-1}$.

$\tau_{1}=\sqrt{\frac{\pi \mu_{\theta \theta}}{\gamma \alpha H_{A}}} \frac{1}{\gamma \alpha H_{A}+\gamma J_{S}} \exp \left(\frac{\gamma \alpha H_{A}\left(1-J_{S} / \alpha H_{A}\right)^{2}}{\mu_{\theta \theta}}\right)$.

We see that the spin-transfer torque reduces the effective barrier height [11], while the fluctuations increase the effective temperature, exponentially reducing the escape time. Because of the uniaxial symmetry $\tau_{2} \rightarrow \tau_{1}$ with $J_{S} \rightarrow-J_{S}$. When a clearly preferred switching direction is required, $\mu_{\theta \theta}$ should be kept smaller than $\gamma J_{S}$. But when current and noise can be switched off immediately after a change in the magnetoresistance signifies that switching has occured, this is no longer a constraint. By combining Eqs. (14), (9), and (8) we can establish an analytical relation between the applied noise power, current and switching time. We use Eq. (13) to evaluate the switching time as a function of current and noise spectral density (see Fig. 3). The model is here at room temperature with parameters typical for real spin valves. The ferromagnet $F_{\text {free }}$ is specified by an anisotropy field $H_{A}=50 \mathrm{mT}$ and total magnetic moment $\mathcal{M}_{S}=10^{-17} \mathrm{~A} \mathrm{~m}^{2}$. The interface resistances are taken to be $1 \Omega$, the polarization of the contacts $p=0.2$, and the damping constant $\alpha=0.02$. A typical noise generator power is $15 \mathrm{~dB}$ higher than the thermal noise power of a $50 \Omega$ resistor at $295 \mathrm{~K}$. This gives a value of $S_{w}=1.0 \times 10^{-20} \mathrm{~J} / \Omega$. Higher noise powers, corresponding to a thermal noise at thousands of kelvins, can be readily generated.

From Fig. 3 we see that by keeping the current fixed and increasing the noise, the switching time can be reduced by orders of magnitude. The contours in the inset to the figure show how the current can be reduced by increasing the noise when the switching time is kept fixed. The power dissipated by the system is proportional to $\left\langle I(t)^{2}\right\rangle$, which 


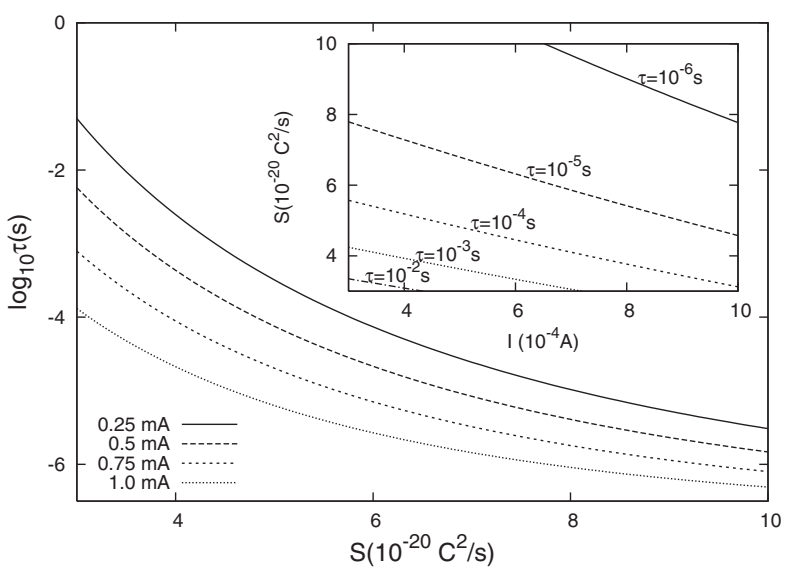

FIG. 3. Switching time as a function of noise spectral density for several values of the current. Inset: Equal-switching-time contours as a function of current and applied noise spectral density.

increases with external noise by $S_{w} \Delta \omega / \pi$. Since the typical bandwidth $\Delta \omega$ is of the order of $1 \mathrm{GHz}$, the main contribution to the power consumption comes from the average switching current. We observe that the power can be reduced by an order of magnitude when a clearly preferred switching direction is necessary and can be reduced even further otherwise.

The calculations presented here are restricted to the high-barrier approximation, which sets limits to the currents and effective temperatures. Still, the mechanism of noise-assisted magnetization switching should be useful in other regimes as well. The experimental realization of this mechanism might face some difficulties such as enabling the high-frequency external fluctuations to reach the system. For instance, in the case of a large environmental capacitance between the leads, the impedance mismatch to the device might require additional measures. Another application of this system would be the measurement of the noise level: by calibration of the device, an unambiguous relation between current, switching time and noise power can be established. We also note that the same mechanism can be used as well to accelerate magneticfield induced switching. An advantage is then that individual bits can be addressed by an unpolarized noisy current, which makes the need for localization of the magnetic field less stringent.

In conclusion, we propose an energy-efficient scheme for current-induced magnetization switching that is assisted by noise. Our approach is based on solving stochastic equations depending on the spectral density of an external noise source. The solution of the corresponding Fokker-Planck equation gives the dependence of the switching time on current and noise level. The current necessary to switch the magnetization can be reduced by applying externally generated current fluctuations. Without importantly complicating the device architecture, the efficiency of spin-transfer torque devices can be improved by exponentially reduced switching times and an order-ofmagnitude smaller power consumption. This could make the difference for the attractiveness of the current-induced switching mechanism for real-life applications.

This work is supported by the "Stichting voor Fundamenteel Onderzoek der Materie" (FOM), and the "Nederlandse Organisatie voor Wetenschappelijk Onderzoek" (NWO). We would like to thank Ya.M. Blanter, A. Brataas, and Yu. V. Nazarov for discussions.

*Present address: Schlumberger Research and Development Inc., Taganskaya Street 9, Moscow, Russia.

[1] Spin Dynamics in Confined Magnetic Structures, edited by B. Hillebrands and K. Ounadjela (Springer, Berlin, 2003); Applications of Magnetic Nanostructures, edited by S. Maekawa and T. Shinjo (Taylor \& Francis, New York, 2002).

[2] J. C. Slonczewski, J. Magn. Magn. Mater. 159, L1 (1996).

[3] L. Berger, Phys. Rev. B 54, 9353 (1996).

[4] F. J. Albert, J. A. Katine, R. A. Buhrman, and D. C. Ralph, Appl. Phys. Lett. 77, 3809 (2000).

[5] J. A. Katine, F. J. Albert, R. A. Buhrman, E. B. Myers, and D. C. Ralph, Phys. Rev. Lett. 84, 3149 (2000).

[6] J. Grollier, V. Cros, A. Hamzic, J. M. George, H. Jaffrès, A. Fert, G. Faini, J. Ben Youssef, and H. Legall, Appl. Phys. Lett. 78, 3663 (2001).

[7] I. N. Krivorotov, N. C. Emley, J. C. Sankey, S. I. Kiselev, D. C. Ralph, and R. A. Buhrman, Science 307, 228 (2005).

[8] A. Brataas, Y. V. Nazarov, and G. E. W. Bauer, Phys. Rev. Lett. 84, 2481 (2000).

[9] M.D. Stiles and A. Zangwill, Phys. Rev. B 66, 014407 (2002).

[10] X. Waintal, E. B. Myers, P. W. Brouwer, and D. C. Ralph, Phys. Rev. B 62, 12317 (2000).

[11] D. M. Apalkov and P.B. Visscher, Phys. Rev. B 72, 180405(R) (2005); D. M. Apalkov and P. B. Visscher, J. Magn. Magn. Mater. 286, 370 (2005).

[12] J. Manschot, A. Brataas, and G. E. W. Bauer, Appl. Phys. Lett. 85, 3250 (2004).

[13] K.-J. Lee, A. Deac, O. Redon, J.-P. Nozières, and B. Dieny, Nat. Mater. 3, 877 (2004).

[14] A. D. Kent, B. Özyilmaz, and E. del Barco, Appl. Phys. Lett. 84, 3897 (2004).

[15] T. Devolder, C. Chappert, P. Crozat, A. Tulapurkar, Y. Suzuki, J. Miltat, and K. Yagami, Appl. Phys. Lett. 86, 062505 (2005).

[16] Z.Z. Sun and X. R. Wang, cond-mat/0511135.

[17] W. F. Brown, Phys. Rev. 130, 1677 (1963).

[18] J. Foros, A. Brataas, Y. Tserkovnyak, and G. E. W. Bauer, Phys. Rev. Lett. 95, 016601 (2005).

[19] Y. Tserkovnyak, A. Brataas, and G.E.W. Bauer, Phys. Rev. Lett. 88, 117601 (2002). 